\title{
Cáncer gástrico en una región de Chile: Comparación de variables clínicas y morfológicas en dos períodos (1986-1995 y 1996-2005)*
}

\author{
Drs. OSCAR TAPIA E. ${ }^{1}$, JUAN CARLOS ROA S. ${ }^{1}$, CARLOS MANTEROLA D. ${ }^{2,3}$, \\ MIGUEL VILLASECA H. ${ }^{1}$, VALENTINA GUTIÉRREZ M. ${ }^{1}$, \\ PLÁCIDO FLORES O. ${ }^{2}$, JUAN CARLOS ARAYA O. ${ }^{1}$, PABLO GUZMÁN G. ${ }^{1}$
}

Departamento de Anatomía Patológica. Facultad de Medicina. Universidad de La Frontera.

Departamento de Cirugía y Traumatología, Facultad de Medicina. Universidad de La Frontera.

3 CIGES (Training, Investigation and Evidence for Based Health Medicine), Universidad de La Frontera. Temuco, Chile.

\begin{abstract}
\section{Changes in morphological an clinical characteristics of gastric cancer} from 1986 to 2005

Background: In the last decade, changes in therapy and on the epidemiological and pathological behavior of gastric cancer have occurred in Chile. Aim: To compare the natural history of gastric cancer in two periods (1986-1995 and 1996-2005) in the ninth region of Chile. Material and Methods: Medical records of 563 gastrectomies for gastric cancer, performed between January 1986 and December 2005 and kept at a Pathology Unit of a public hospital, were reviewed. Clinical and morphological variables were analyzed (histological Lauren type, differentiation degree, location and macroscopic type, infiltration level, number of resected and involved lymph nodes). Results: A significant increase in the frequency of the diffuse type of gastric cancer was observed in the second period in study, with a concomitant decrease of the intestinal type. A reduction in tumor size and number of excised lymph nodes was also observed. Conclusions: In the second study period, morphological changes in gastric cancer, were observed.
\end{abstract}

Key words: Gastric cancer, epidemiology, natural history.

\section{Resumen}

Introducción: El cáncer gástrico $(\mathrm{CG})$ es la primera causa de muerte por cáncer en Chile (tasa de mortalidad general de $19,5 \times 10^{5}$ y $24,8 \times 10^{5}$ habitantes a nivel nacional y IX Región respectivamente). Se han descrito cambios epidemiológicos, histopatológicos y de tratamiento para CG. El objetivo de este estudio es describir la historia natural del CG en la IX región de Chile comparando dos periodos (1986-1995 y 19962005). Material y Método: Cohorte histórica. Se incluyeron todos los casos de gastrectomías por CG de la

\footnotetext{
*Recibido el 3 de Septiembre de 2009 y aceptado para publicación el 17 de Noviembre de 2009.

Correspondencia: Dr. Oscar Tapia E.

Manuel Montt 112, Código Postal 478-1176, Temuco, Chile.

E-mail: otapi001@pinhue.ufro.cl
}

Financiado en parte por la Dirección de investigación de la Universidad de La Frontera. DIUFRO 120528. 
Unidad de Anatomía Patológica del Hospital Hernán Henríquez Aravena entre Enero de 1986 y Diciembre de $2005(\mathrm{n}=563)$. Se estudiaron variables clínicas (edad, género y estadio TNM) y morfológicas (tipo histológico según Lauren, grado de diferenciación, localización y tipo macroscópico de la lesión, nivel de infiltración, número de ganglios resecados y comprometidos por el tumor). Se aplicó estadística descriptiva y analítica usando t-test y ANOVA para variables continuas, chi $^{2}$ y exacto de Fisher para variables categóricas. Resultados: De las características estudiadas, se evidenció un incremento estadísticamente significativo del tipo difuso en el segundo período en estudio a expensas de un decremento del tipo intestinal; así también una disminución en el tamaño tumoral y número de ganglios linfáticos resecados. Conclusiones: Se verificaron cambios morfológicos del CG experimentados a nivel regional en los períodos estudiados.

Palabras clave: Cáncer gástrico, epidemiología, historia natural.

\section{Introducción}

El cáncer gástrico $(C G)$ es la primera causa de muerte por cáncer en Chile, con una tasa de mortalidad general de $19,5 \times 10^{5}$ habitantes y de 25,3 x $10^{5}$ en hombres; constituyendo el tumor maligno más frecuente en hombres. En la IX región la tasa de mortalidad general alcanza a $24,8 \times 10^{5}$ habitantes y en hombres a $33,7 \times 10^{5}$. Si bien en las últimas tres décadas se ha observado una estabilización en la tasa de mortalidad general por este tumor, hoy en día la probabilidad media de que un chileno muera por CG es de aproximadamente 3\%, haciendo de esta enfermedad un problema de salud pública ${ }^{1-3}$. Por esta razón, es que en nuestro país se han creado políticas nacionales de salud destinadas a mejorar el diagnóstico y realizar tratamiento en tiempos garantizados; y así disminuir la tasa de mortalidad de esta enfermedad, potencialmente curable en estadios precoces $^{4,5}$.

Está ampliamente documentado que el CG es más frecuente en países subdesarrollados y en vías de desarrollo afectando mayoritariamente a hombres, con una incidencia pico en la $6^{\text {a }}$ década de la vida ${ }^{6-9}$. En los últimos años se han descrito cambios epidemiológicos, morfológicos y terapéuticos en relación al CG como en relación a localización tumoral, tipo histológico según Laurén, nivel de infiltración (incipiente o avanzado), estadio TNM, tipo de resecciones realizadas y morbimortalidad operatoria asociada. Se ha descrito también, un incremento progresivo en la frecuencia del tipo difuso según Laurén llegando en algunos países a superar al tipo intestinal, esto último en estrecha relación con la localización tumoral, reportándose actualmente una mayor frecuencia de tumores ubicados en el tercio superior y cardial; condición descrita como esperable en países desarrollados mientras que tumores de tercio inferior son más frecuentes en países en vías de desarrollo ${ }^{7,9,10-13}$. Del mismo modo, se ha descrito un aumento gradual en el diagnóstico de lesiones incipientes a través de los años con el consiguiente impacto en la supervivencia de estos pacientes, condición determinada principalmente por los pro- gramas de detección precoz instaurados en Japón, China y Corea; el mejoramiento tecnológico de equipos; y la capacitación continua de endoscopistas y patólogos ${ }^{14-17}$.

Los cambios descritos estarían influenciados por múltiples factores entre los que se mencionan modificación de hábitos alimentarios con aumento en el consumo de vegetales y menor consumo de carnes y productos salados; uso masivo de la refrigeración de alimentos e implementación de tratamientos altamente efectivos en la erradicación del Helicobacter pylori ${ }^{10,11,18-23}$.

Este artículo se ha escrito siguiendo la propuesta STROBE, para el reporte de estudios observacionales ${ }^{24}$.

La pregunta de investigación de este estudio es ¿existen diferencias en algunas variables clínicas y morfológicas de pacientes con $\mathrm{CG}$ en un período de 20 años al comparar dos decenios en una región de Chile de alta prevalencia e incidencia de la enfermedad?

El objetivo de este trabajo es comparar el comportamiento de algunas variables clínicas y morfológicas de pacientes con CG en un período de 20 años considerando para ello dos decenios (1986-1995 y 1996-2005), en una región de Chile de elevada prevalencia e incidencia de la enfermedad.

\section{Material y Método}

Diseño del estudio: Cohorte histórica.

Marco: Se recogieron los datos provenientes de pacientes diagnosticados de CG en la Unidad de Anatomía Patológica del Hospital Hernán Henríquez Aravena de la ciudad de Temuco entre enero de 1986 y diciembre de 2005.

Participantes: Se incluyeron todos los casos de gastrectomías por CG diagnosticados de forma consecutiva en el período e institución antes señalados. No se excluyó ningún caso. El seguimiento de los casos se realizó mediante el estudio de las historias clínicas y certificados de defunción obtenidos del Registro Civil e Identificación, desde el momento 
del diagnóstico hasta al menos tres años posterior a este.

Variables de interés: Se consideró la edad, género; el tipo histológico según Lauren ${ }^{(1)}$ (dicotomizado en intestinal y difuso); el grado de diferenciación histológica (categorizado en bien diferenciado, moderadamente diferenciado y poco diferenciado), prevaleciendo para su categorización el menor grado de diferenciación presente en el tumor; la localización macroscópica de la lesión (categorizado en tercios superior o cardial, medio e inferior o antral, según clasificación japonesa para estudio del CG), revisándose para ello, archivos fotográficos y digitales para determinar la probable localización original del tumor en aquellas lesiones en las que se verificó compromiso de más de un segmento ${ }^{26}$; el tipo macroscópico (categorizado según la clasificación macroscópica de la sociedad japonesa de gastroenterología endoscópica para tumores incipientes y clasificación de Bormann para tumores avanzados); el nivel de infiltración (categorizado según el compromiso de la pared gástrica en tumores incipientes [intramucoso y submucoso] y avanzados [muscular propia, subserosa y serosa]); el número de ganglios resecados; y el número de ganglios comprometidos por el tumor.

Sesgos: Se minimizó el sesgo de clasificación mediante la comprobación de los datos por investigadores independientes con amplia experiencia en el tema (JCR, MV, JCA y PG).

Tamaño de la muestra: No se realizó estimación de tamaño de la muestra pues se trabajó con la totalidad del universo de pacientes con $\mathrm{CG}$ en el período señalado.

Tabla 1. Distribución de algunas variables clínicas y morfológicas por periodos

\begin{tabular}{|c|c|c|c|}
\hline Variables & $\begin{array}{c}1986-1995 \\
(n=264)\end{array}$ & $\begin{array}{c}1996-2005 \\
(n=299)\end{array}$ & $\mathbf{p}$ \\
\hline Género (\%) & & & 0,96 \\
\hline Femenino & $33,0 \quad(87)$ & $33,0 \quad(99)$ & \\
\hline Masculino & $67,0(177)$ & $67,0(200)$ & \\
\hline Edad (años) & $60,1 \pm 11,3$ & $61,2 \pm 12,2$ & \\
\hline Resección Quirúrgica (\%) & & & 0,24 \\
\hline Gastrectomía subtotal & $47,0(124)$ & $52,0(155)$ & \\
\hline Gastrectomía total & $53,0(140)$ & $48,0(144)$ & \\
\hline Localización (\%) & & & 0,37 \\
\hline $1 / 3$ superior & $28,0 \quad(74)$ & $31,0 \quad(93)$ & \\
\hline $1 / 3$ medio & 28,0 & 28,0 & \\
\hline $1 / 3$ inferior & $44,0(116)$ & $41,0(122)$ & \\
\hline Tipo Macroscópico (\%) & & & 0,20 \\
\hline Cáncer Incipiente & $13,0 \quad(34)$ & $17,0 \quad(50)$ & \\
\hline Tipo I & $21,0 \quad(7)$ & $16,0 \quad(8)$ & \\
\hline Tipo II & 70,0 & $60,0 \quad(30)$ & \\
\hline Tipo III & $9,0 \quad(3)$ & $24,0 \quad(12)$ & \\
\hline Cáncer Avanzado & $87,0(230)$ & $83,0(249)$ & \\
\hline Bormann 1 & $7,0 \quad(16)$ & $5,0 \quad(12)$ & \\
\hline Bormann 2 & $24,0 \quad(55)$ & $12,0 \quad(30)$ & \\
\hline Bormann 3 & $37,0 \quad(85)$ & $50,0(125)$ & \\
\hline Bormann 4 & $26,0 \quad(60)$ & $27,0 \quad(67)$ & \\
\hline Bormann 5 & $6,0 \quad(14)$ & $6,0 \quad(15)$ & \\
\hline Tamaño Tumoral (mm) & $65,8 \pm 33,3$ & $55,8 \pm 31$ & 0,0002 \\
\hline Número de ganglios resecados & $32,6 \pm 17,6$ & $29 \pm 15,3$ & 0,005 \\
\hline Número de ganglios positivos & $7,15 \pm 2,3$ & $5,9 \pm 1,7$ & 0,06 \\
\hline
\end{tabular}


Tabla 2. Distribución de variables morfológicas por periodos

\begin{tabular}{|c|c|c|c|}
\hline Variables & $\begin{array}{c}1986-1995 \\
(n=264)\end{array}$ & $\begin{array}{c}1996-2005 \\
(n=299)\end{array}$ & $\mathbf{p}$ \\
\hline Grado de Diferenciación (\%) & & & 0,83 \\
\hline Bien diferenciado & $8,0 \quad(21)$ & $9,0 \quad(27)$ & \\
\hline Moderadamente diferenciado & $35,0 \quad(92)$ & $30,0 \quad(90)$ & \\
\hline Poco diferenciado & $57,0(151)$ & $61,0(182)$ & \\
\hline Tipo Histológico de Lauren (\%) & & & 0,0001 \\
\hline Intestinal & $54,0(143)$ & $38,0(114)$ & \\
\hline Difuso & $46,0(121)$ & $62,0(185)$ & \\
\hline Infiltración (\%) & & & 0,72 \\
\hline Mucosa & $7,0 \quad(18)$ & $6,0 \quad(18)$ & \\
\hline Submucosa & $6,0 \quad(16)$ & $11,0 \quad(32)$ & \\
\hline Muscular propia & $11,0 \quad(29)$ & $12,0 \quad(36)$ & \\
\hline Subserosa & $39,0(103)$ & $34,0(102)$ & \\
\hline Serosa & $37,0 \quad(98)$ & $37,0(111)$ & \\
\hline Estadio TNM (\%) & & & 0,96 \\
\hline I & $23,0 \quad(61)$ & $29,0 \quad(87)$ & \\
\hline II & $27,0 \quad(71)$ & $19,0 \quad(57)$ & \\
\hline III & $42,0(111)$ & $42,0(125)$ & \\
\hline IV & $8,0 \quad(21)$ & $10,0 \quad(30)$ & \\
\hline
\end{tabular}

Métodos estadísticos: Utilizando los paquetes estadísticos Epi-info 6.0 y Stata 9.0, se realizó un análisis exploratorio de los datos y posteriormente se aplicó estadística descriptiva con cálculo de promedios y desviaciones estándar, medianas y valores extremos para variables continuas; cálculo de porcentajes para variables categóricas; y de estadística analítica, utilizando t-test y análisis de la varianza (ANOVA) para variables continuas, $\mathrm{Chi}^{2}$ de Pearson y exacto de Fisher para variables categóricas.

\section{Resultados}

El grupo en estudio considera 563 sujetos gastrectomizados por $\mathrm{CG}$, todos los cuales concluyeron el período de seguimiento mínimo definido en la metodología (1986 y 2005); correspondiendo 264 casos (47\% de la muestra total) a los reclutados en el período $1986-1995$ y 299 casos (53\% de la muestra total) a los reclutados en el período 1996-2005 respectivamente.

El $67 \%$ del grupo total era de género masculino y la edad promedio de este fue de $60,7 \pm 11,7$ años. No se verificaron diferencias estadísticamente significativas al comparar las variables edad, género, localización de la lesión, tipo de resección gástrica realizada y tipo macroscópico del tumor (Tabla 1). Se evidenció que en el segundo período en estudio el tamaño tumoral y número de ganglios linfáticos resecados fue estadísticamente menor que en el primer período en evaluación (Tabla 1).

No se constataron diferencias estadísticamente significativas al comparar las variables grado de diferenciación, nivel de infiltración y estadío TNM en los grupos en estudio. Sólo se evidenció diferencias estadísticamente significativas al comparar la variable tipo histológico de Lauren; observándose un incremento del tipo difuso en el segundo período en estudio a expensas de un decremento del tipo intestinal (Tabla 2).

\section{Discusión}

Se trata de un estudio en el que se intentó identificar cambios clínico-morfológicos del CG en una población de alta prevalencia e incidencia de la enfermedad, a través de un estudio de cohorte retrospectiva; diseño que aporta un nivel de evidencia $2 b$ para este tipo de escenarios, según la clasificación actualizada del Centro de Medicina Basada en la Evidencia de la Universidad de Oxford ${ }^{27}$. 
El CG constituye en nuestro país un problema de salud pública, afectando predominantemente a hombres en edad económicamente productiva y en una proporción hombre/mujer de 2:1,2,5,9,28, siendo además estos tumores diagnosticados generalmente en etapas avanzadas con la consiguiente menor supervivencia y mayor morbilidad ${ }^{5,29-31}$.

En nuestro estudio, al igual que lo publicado por otros autores, observamos un aumento en el número de lesiones incipientes diagnosticadas $(13 \%$ a $17 \%$ ), sin embargo, tumores avanzados continúan siendo los más frecuentes; situación muy distinta a la de países como Japón y Corea, donde la aplicación efectiva de programas de tamizaje permiten detectar aproximadamente 1 caso por cada 800 pacientes examinados, correspondiendo la mitad de ellos a lesiones incipientes ${ }^{16,32,33}$. Si bien estamos lejos de cifras como las observadas en Japón para lesiones incipientes; Chile cuenta con políticas de salud orientadas ha aumentar la pesquisa en etapas precoces en grupos de riesgo y pacientes sintomáticos. Es así como estudios nacionales realizados con anterioridad, han demostrado que es posible detectar aproximadamente un caso de CG por cada 40-50 procedimientos endoscópicos cuando el tamizaje se focaliza en adultos mayores de 40 años, sintomáticos; siendo alrededor de un $20 \%$ de los CG detectados de esta forma, del tipo incipiente i, $^{5,34}$.

En los dos períodos estudiados, los tumores localizados en el tercio inferior fueron los más frecuentes (44\% y 41\%), aunque se observó un incremento progresivo en el tiempo de tumores proximales (28\% a $31 \%)$; lo que coincide con la situación morfológica del CG descrita para países en vías de desarrollo como es nuestro país s, $^{8,33,30,35,36}$.

En dos series previamente publicadas ${ }^{34,37}$, se ha descrito una baja en los tumores de la variedad intestinal de Lauren, con aumento del tipo difuso; situación que se verificó también en nuestro estudio; con un aumento estadísticamente significativo de $46 \%$ a $55 \%$.

El menor número de ganglios comprometidos por tumor y el menor tamaño encontrado en los tumores estudiados en la década 1996-2005 estaría asociado al aumento en el número de lesiones incipientes diagnosticadas. El mayor número de ganglios linfáticos resecados en el período 1986-1995 es explicado por el mayor número de gastrectomías totales realizadas en este período en comparación con la posterior década ( $53 \%$ y $48 \%$ ) y consiguiente mayor recuento ganglionar en gastrectomías totales que subtotales (35,5 y 25,9 ganglios respectivamente).

Si bien observamos tendencias al comparar variables morfológicas de los tumores, no encontramos diferencias significativas en cuanto a la localización y nivel de infiltración (incipiente/avanzado). Este hecho podría estar dado por la superposición temporal de casos con características intermedias. Con este objetivo y de manera de verificar las tendencias para estas variables, comparamos casos de los quinquenios extremos (1986-1990 y 2001-2005), encontrando un aumento estadísticamente significativo de los tumores localizados en tercio superior ( $45 \%$ a $51 \%$ ) $(\mathrm{p}<0,05)$ y no observando diferencias estadísticas según el nivel de infiltración.

Si bien se constató un incremento en el número de lesiones gástricas incipientes diagnosticadas, hoy el CG continúa siendo la primera causa de muerte por cáncer en Chile, a pesar de la modificación de factores de riesgo logrados por el mejoramiento tanto de las condiciones socio-económicas como ambientales. Es posible que las políticas de salud vigentes en nuestro país desde julio de 2006, tendientes a identificar grupos de riesgo y lesiones en etapas precoces, asociado al continuo y creciente progreso como país, permitirán en los próximos años evidenciar los cambios epidemiológicos de esta enfermedad ya experimentados en países como Japón y Corea; en los que ha aumentado significativamente la pesquisa de lesiones incipientes y por tanto reducido la mortalidad por CG.

\section{Referencias}

1. Medina E, Kaempfer AM. Cancer mortality in Chile: epidemiological considerations. Rev Med Chile 2001; 129: 1195-202.

2. http://deis.minsal.cl/ev/mortalidad_causas/cancer_estomago/series/consulta.asp. Ministerio de Salud de Chile.

3. de Aretxabala X, Araya JC, Flores P, Roa I, Fernández E, Wistuba I, y cols. Características del cáncer gástrico en la IX región de Chile. Rev Med Chile 1992; 120: 407-414.

4. Estudio del análisis de la información científica para el desarrollo de protocolos AUGE: Cáncer gástrico. Universidad de Chile. Escuela de Medicina. Escuela de Salud Pública, abril 2004.

5. Guías clínicas GES 2006 para Cáncer Gástrico, Ministerio de Salud-Chile.

6. Csendes A, Smok G, Medina E, Salgado I, Rivera R, Quitral M. Características clínicas del cáncer gástrico 1958-1990. Rev Med Chile 1992; 120: 36-42.

7. Roder DM. The epidemiology of gastric cancer. Gastric Cancer. 2002; 5 Suppl 1: 5-11.

8. Crew KD, Neugut AI. Epidemiology of gastric cancer. World J Gastroenterol 2006; 12: 354-362.

9. Calderón ME, Csendes A, Ospina C. Cambios en la localización y en el tipo patológico del cáncer gástrico en el período 1975-2005. Rev Chil Cir 2007; 59: 366-369.

10. Chan AO, Wong BC, Lam SK. Gastric cancer: past, present and future. Can J Gastroenterol 2001; 15: 469-474. 
11. Bulanov D. Gastric cancer-current state of the problem. Part I. Epidemiology. Pathology. Classification. Staging. Khirurgiia (Sofiia) 2007; 5: 45-52.

12. Wang X, Wu CX, Zheng Y, Wang JJ. Time trends and characteristics of gastric cancer incidence in urban Shanghai. Zhonghua Liu Xing Bing Xue Za Zhi 2007; 28: 875-880.

13. Liu Y, Kaneko S, Sobue T. Trends in reported incidences of gastric cancer by tumour location, from 1975 to 1989 in Japan. Int J Epidemiol 2004; 33: 808-815.

14. Hallissey MT, Allum WH, Jewkes AJ, Ellis DJ, Fielding JW. Early detection of gastric cancer. BMJ 1990; 301: 513-515.

15. Eckardt VF, Giessler W, Kanzler G, Remmele W, Bernhard G. Clinical and morphological characteristics of early gastric cancer. A case-control study. Gastroenterology 1990; 98: 708-714.

16. Hisamichi S. Screening for gastric cancer. World J Surg 1989; 13: 31-37.

17. Csendes A, Smok G, Velasco N, Godoy M, Medina E, Braghetto I, y cols. Cáncer gástrico incipiente e intermedio. Análisis clínico y sobrevida de 51 casos. Rev Med Chile 1980; 108: 1011-1015.

18. Brenner H, Rothenbacher D, Arndt V. Epidemiology of stomach cancer. Methods Mol Biol 2009; 472: 467-477.

19. Neugut AI, Hayek M, Howe G. Epidemiology of gastric cancer. Semin Oncol 1996; 23: 281-291.

20. Terry MB, Gaudet MM, Gammon MD. The epidemiology of gastric cancer. Semin Radiat Oncol 2002; 12: 111-127.

21. Tredaniel J, Boffetta P, Buiatti E, Saracci R, Hirsch A. Tobacco smoking and gastric cancer: review and metaanalysis. Int J Cancer 1997; 72: 565-573.

22. Van den Brandt PA, Botterweck AA, Goldbohm RA. Salt intake, cured meat consumption, refrigerator use and stomach cancer incidence: a prospective cohort study (Netherlands). Cancer Causes Control 2003; 14: 427-438.

23. Helicobacter and Cancer Collaborative Group. Gastric cancer and Helicobacter pylori: a combined analysis of 12 case control studies nested within prospective cohorts. Gut 2001; 49: 347-353.

24. Von Elm E, Altman DG, Egger M, Pocock SJ, Gøtzsche PC, Vandenbroucke JP; STROBE Initiative. The Strengthening the Reporting of Observational Studies in Epidemiology (STROBE) statement: guidelines for reporting observational studies. J Clin Epidemiol 2008; 61: 344-349.

25. Lauren $\mathrm{P}$. The two histological main types of gastric carcinoma: diffuse and so-called intestinal type carcinoma. Acta Path Microbiol Scan 1965; 64: 31-49.

26. Japanese Research Society for Gastric Cancer. The General Rules for the Gastric Cancer study in Surgery and Pathology. Jap J Surg 1981; 11: 27-25.

27. Centre for Evidence Based Medicine (CEBM). Oxford Centre for Evidence-based Medicine-Levels of Evidence (March 2009). Available from: http://www.cebm. net/index.aspx?o=1025. Visited June 222009.

28. Armijo R. Descriptive epidemiology of cancer in Chile: 1973-78. Nati Cancer Inst Monogr 1982; 62: 91-94.

29. Calvo Belmar A, Pruyas M, Nilsen E, Verdugo P. Estudio de cáncer gástrico en pacientes sintomáticos digestivos 1996 a 2000. Rev Med Chile 2001; 129: 749-755.

30. Buonadonna A, Lombardi D, De Paoli A, Bidoli E, Frustaci S. Adenocarcinoma of the stomach: univariate and multivariate analyses of factors associated with survival. Tumori 2003; Suppl 2 (5): S31-S34.

31. Green D, Ponce de Leon S, León-Rodríguez E, SosaSánchez R. Adenocarcinoma of the stomach: univariate and multivariate analysis of factors associated with survival. Am J Clin Oncol 2002; 25: 84-89.

32. Tsubono Y, Nishino Y, Tsuji I, Hisamichi S. Screening for Gastric Cancer in Miyagi, Japan: Evaluation with a Population-Based Cancer Registry. Asian Pac J Cancer Prev 2000; 1: 57-60.

33. Mizoue T, Yoshimura T, Tokui N, Hoshiyama Y, Yatsuya H, Sakata K, et al; Japan Collaborative Cohort Study Group. Prospective study of screening for stomach cancer in Japan. Int J Cancer 2003; 106: 103-107.

34. Hoffemberg P, Bahamondes G, López H, Cozzi V, Rufin F, Dávila M y cols. Pesquisa endoscópica de lesiones gástricas en voluntarios aparentemente sanos. Rev Med Chile 1978; 106: 586-590.

35. Inoue M, Tsugane S. Epidemiology of gastric cancer in Japan. Postgrad Med J 2005; 81: 419-424.

36. Crew KD, Neugut AI. Epidemiology of Gastric Cancer. World J Gastroenterol 2006; 12: 354-362.

37. Kaneko S, Yoshimura T. Time trend analysis of gastric cancer incidence in Japan by histological types, 19751989. Br J Cancer 2001; 84: 400-405. 